\title{
Delayed implantation in giant pandas: the first comprehensive empirical evidence
}

\author{
Hemin Zhang, Desheng Li, Chendong Wang and Vanessa Hull ${ }^{1}$ \\ China Conservation and Research Center for the Giant Panda (CCRCGP), Wolong Nature Reserve, Sichuan 623006, \\ People's Republic of China and ${ }^{1}$ Department of Fisheries and Wildlife, Center for Systems Integration and \\ Sustainability (CSIS), Michigan State University, East Lansing, Michigan 48823, USA
}

Correspondence should be addressed to D Li; Email: lidesheng18060@yahoo.com.cn

\begin{abstract}
Successful conservation of an endangered species relies on a good understanding of its reproductive biology, but there are large knowledge gaps. For example, many questions remain unanswered with regard to gestation and fetal development in the giant panda. We take advantage of a sample size that is unprecedented for this species $(n=13)$ to explore patterns in reproductive development across individuals at the China Conservation and Research Center for the Giant Panda. We use ultrasound techniques on multiple giant pandas for the first time to empirically confirm what has long been suspected that pandas exhibit delayed implantation of the embryo. We also show that the duration of postfetal detection period is remarkably similar across individuals $(16.85 \pm 1.34$ days $)$. Detection of fetus by ultrasound was strongly correlated to the peak in urinary progesterone $(r=0.96, t=8.48, d . f .=8, P=0.0001)$ and swelling in the mammary glands $(r=0.79, t=3.61$, d.f. $=8, P=0.007)$ and vulva $(r=0.91, t=6.40$, d.f. $=8, P=0.0002)$ of adult females. When controlling for both the duration of the total gestation period and the postfetal detection period, infant birth weight was only significantly predicted by the latter $(\beta=11.25$, S.E.M. $=4.98, t=2.26, P=0.05)$, suggesting that delayed implantation increases flexibility in the timing of birth but is not important in dictating infant growth. This study informs reproductive biology by exploring the little-studied phenomenon of delayed implantation in relationship to physiological changes in pregnant giant panda females.

Reproduction (2009) 138 979-986
\end{abstract}

\section{Introduction}

Studies on gestation and fetal development are important for understanding reproduction of animals, particularly wildlife species threatened with extinction. One such species is the giant panda (Ailuropoda melanoleuca), an endangered mammal and global conservation icon endemic to China. Human threats to the wild giant pandas and their habitat have inspired the establishment of captive giant panda-breeding programs with the hope of one day releasing captive animals to the wild. Captive giant panda breeding was difficult for a long time since its beginning in 1978 (Schaller et al. 1985), but recently prospered due to advances in artificial insemination, infant care, and captive animal management (Zhang et al. 2004). As of 2006, more than 260 giant pandas have been bred in captivity worldwide (Ballou et al. 2006). Such advances in captive breeding provide new opportunities to examine the development of giant pandas at their earliest stages, beginning with the fetus.

While there has been extensive research on the detection of estrus and mating of giant pandas in captivity (Lindburg et al. 2001, McGeehan et al. 2002,
Zhang et al. 2004) in addition to infant care and development (Zhang et al. 1996, Zhu et al. 2001), little has been reported on the crucial stage between these two - development of the giant panda fetus and changes in the pregnant mother's physiology. The reason for this is perhaps that pregnancy has been notoriously difficult to detect in giant pandas in part because they gain little weight and their fetuses develop late (Sutherland-Smith et al. 2004). In the past, captive managers were often unable to detect a female's pregnancy status until days before the birth, with pseudopregnancies often reported.

Despite this challenge, basic information that has been documented includes the observation that wild giant pandas have gestation lengths of 97-161 days (Schaller et al. 1985). This is a long gestation time when considering the small, young, altricial giant panda that weigh a mere $90-130 \mathrm{~g}$ (Schaller et al. 1985). The reason is that like other ursids, giant pandas may exhibit a phenomenon known as delayed implantation, in which the embryo floats in the womb and arrests development until it attaches to the uterus months later. Delayed implantation is believed to occur in giant pandas based on hormone analyses (Hodges et al. 1984, 
Chaudhuri et al. 1988) and the observed discrepancy between the panda's long gestation coupled with underdeveloped young (Zhu et al. 2001). This physiological response is believed to be seasonal and could be an adaptation to an unpredictable food source that would make it advantageous for the birth of the offspring to match a time of highest availability of food sources (Mead 1989, Sandell 1990). One ultrasound study on a single animal suggested that delayed implantation might occur in giant pandas (Sutherland-Smith et al. 2004), but this has not yet been confirmed in other pandas.

Recent advances in ultrasound technology provide the opportunity of monitoring the development of a giant panda fetus in vivo in a way that has not been possible before. Ultrasound has been used in a small number of cases to monitor reproductive health of giant panda males and females (Hildebrandt et al. 2006); however, there is only one documented case of the use of ultrasound in one pregnancy event (Sutherland-Smith et al. 2004). We set out to fill this gap in the literature by using ultrasound to characterize giant panda fetal development in 13 giant panda pregnancies. In this paper, we describe fetal development and female physiological changes during pregnancy. We also examine relationships between pregnant female urinary progesterone levels, gestation length, timing of fetus detection, and infant birth weight. Furthermore, we discuss implications of the findings for the management of captive giant pandas and the growing understanding of giant panda physiology and evolution.

\section{Results}

\section{Physiological changes in pregnant females}

The first sign of pregnancy in female giant pandas occurred $\sim 3$ months after insemination. Swelling occurred in the mammary glands and vulva 102.00 \pm 18.34 and $96.30 \pm 16.77$ days after insemination respectively (both $N=10$ ). The peak in urinary progesterone occurred $12.75 \pm 4.89$ days before the fetus was detected (Table 1). The magnitude of the peak progesterone level was $206.14 \pm 54.73 \mathrm{ng} / \mathrm{mg}$ creatinine (Cr; ranging from 171.0 to $328.16 \mathrm{ng} / \mathrm{mg} \mathrm{Cr}$ ). There was some degree of variability in the timing and the pattern of change in urinary progesterone among pregnant females across time, although in most females there was a discernable increase to a peak level, beginning around 40-55 days prior to parturition (Fig. 1). The peak was reached $29.25 \pm 5.12$ days before parturition and was followed by an immediate decline in the course of a few days (Fig. 1). The exception to this was Youyou (pregnant in 2006), whose progesterone concentration vacillated at a peak level for a period of around 2 weeks before declining.

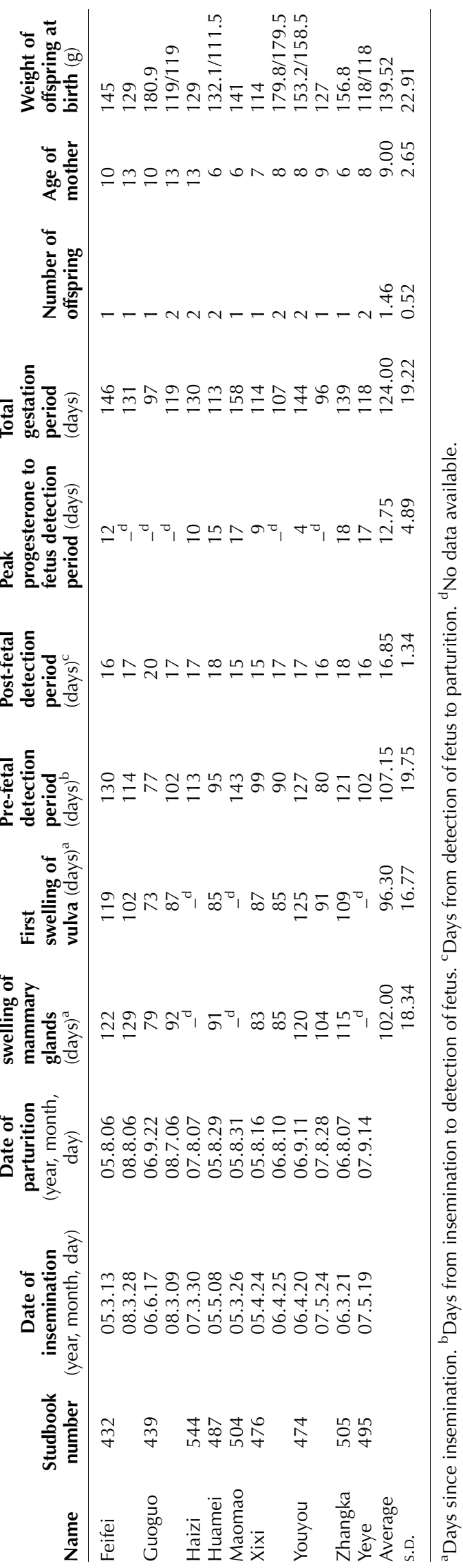



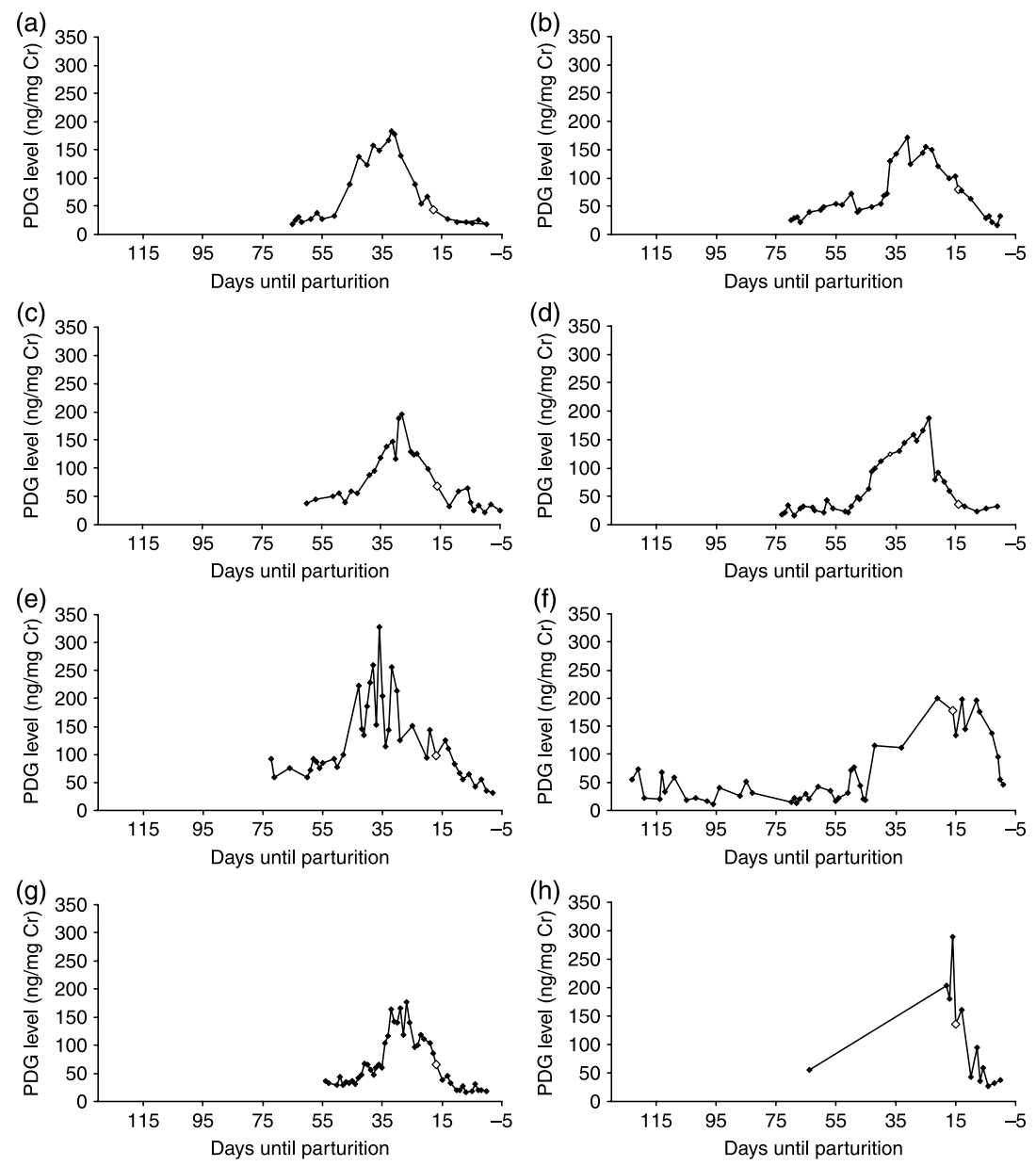

Figure 1 Pregnanediol-3-glucuronide (PDG) level measured using EIA and indexed using creatinine ( $\mathrm{ng} / \mathrm{mg} \mathrm{Cr}$ ) for eight pregnant adult female giant pandas at the China Conservation and Research Center for the Giant Panda (CCRCGP): (a) Huamei 2005, (b) Maomao 2005, (c) Feifei 2005, (d) Xixi 2005, (e) Zhangka 2006, (f) Youyou 2006, (g) Haizi 2007, and (h) Yeye 2007. Open diamonds represent the time of detection of the fetus using ultrasound imaging.

\section{Development of giant panda fetuses}

The giant panda fetuses were detected in a relatively narrow window of 15-20 days before the adult females gave birth $(16.85 \pm 1.34$ days, Table 1$)$. The timing of detection of the fetus was significantly correlated to the start of swelling in the mammary glands $(r=0.79$, $t=3.61$, d.f. $=8, P=0.007)$, swelling in the vulva $(r=0.91$, $t=6.40$, d.f. $=8, P=0.0002$ ), and the peak in urinary progesterone $(r=0.96, t=8.48, \mathrm{~d} . \mathrm{f} .=8, P=0.0001)$.

Out of the 13 pregnancy events, 7 resulted in single births and 6 in twins. The development of the fetus over time is shown in Fig. 2(a-f). The giant panda fetus was so small that the heartbeat could not be detected through ultrasound until the $3 \mathrm{rd}$ or 4th day after detection of the fetus. After 3 or 4 days, the shape of a fetus appeared; however, no differentiation of organs was detected. After 6 or 7 days, the head and the truncus appeared specialized. After 8 or 9 days, the limbs and tail were also visible. At this point, the developing fetus became active by displaying movement of its extremities. With respect to the two individuals for which we were able to reliably record fetal length over time, we found that at days 7 and 9 since the first fetal detection ( 11 days to parturition), the lengths of the fetuses were only 1.7 and $2.9 \mathrm{~cm}$ respectively (Table 2 ). Five days later (6 days to parturition), the fetuses had grown by $311 \%(7.4 \mathrm{~cm})$ and $154 \%(6.6 \mathrm{~cm})$ respectively. The last recorded measurements for fetal length were 10.1 and $11.6 \mathrm{~cm}$.

\section{Gestation length and birth weight}

The female giant panda gestation period ranged from 96 to 158 days $(124.00 \pm 19.22$ days, Table 1$)$. There did not appear to be a strong consistency in the duration of gestation within the same individual across years. For example, although one individual 'Xixi' had similar gestation lengths of 107 and 114 days in two different years, another individual 'Youyou' had a relatively long gestation period of 144 days for 1 year and then a relatively short gestation period of 96 for the next year.

The prefetal detection period was $107.15 \pm 19.75$ days, indicating a period of delayed implantation of the embryo. The duration of the postfetal detection period was not significantly correlated to the duration of the 
(a)

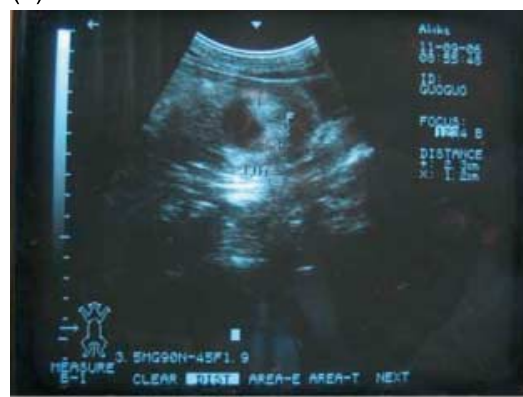

11 days to parturition

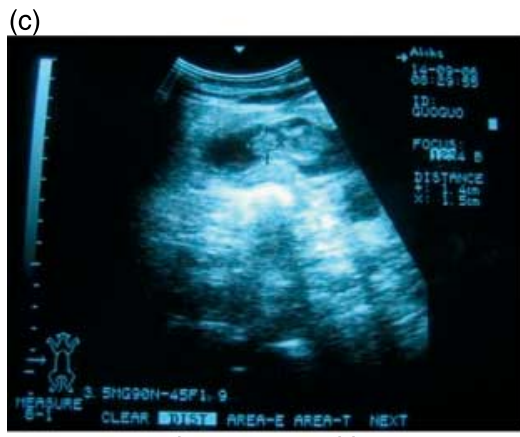

8 days to parturition

(e)

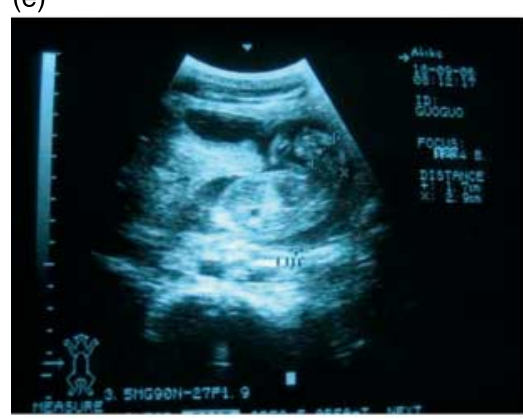

4 days to parturition (b)

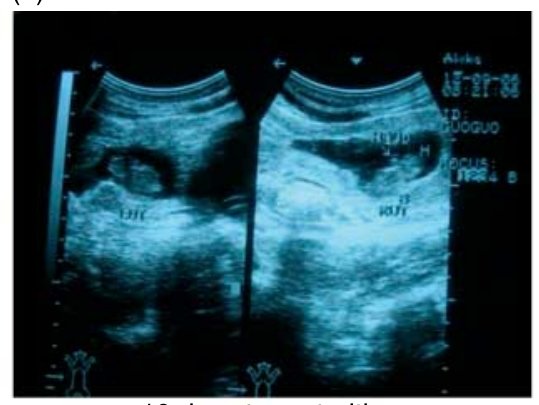

10 days to parturition

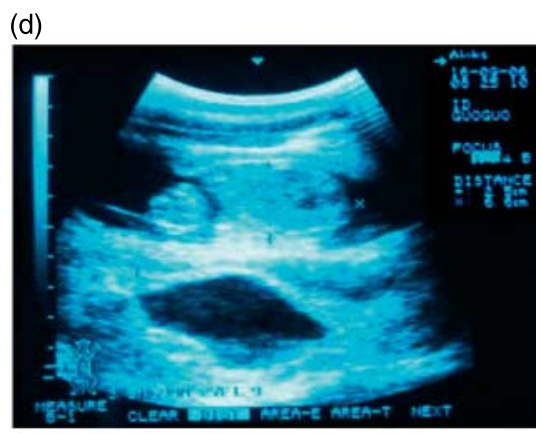

6 days to parturition

(f)

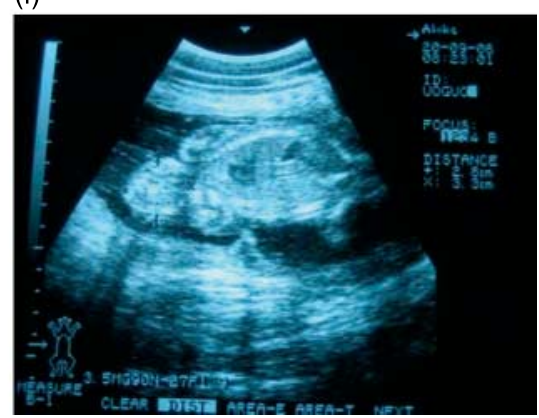

2 days to parturition
Figure 2 (a-f) Ultrasound images depicting the development of the giant panda fetus from detection to near birth. Images are drawn from ultrasound imaging (Aloka SSD-900V ultrasound machine, 3.5 sector transducer) of a pregnant female named Guoguo in 2006 at the China Conservation and Research Center for the Giant Panda (CCRCGP). prefetal detection period $(t=-1.5467$, d.f. $=11$, $P=0.15)$. The average birth weight of infants was $139.52 \pm 22.91 \mathrm{~g}$. Birth weight (averaged in the case of twins) was significantly positively related to the length of the postfetal detection period $(\beta=11.25$, S.E.M. $=4.98$, $t=2.26, P=0.05)$, but not the overall length of gestation, age of the mother, or number of offspring born (all $P>0.4$ ).

\section{Discussion}

\section{Characterizing delayed implantation in giant pandas}

The observations of 13 panda pregnancies using ultrasound in this study convincingly confirm that giant pandas exhibit delayed implantation. It is well known that the ursid fetus is too small to be detected via ultrasound until some time after implantation of the embryo on the uterus occurs (Tsubota et al. 1987,
Göritz et al. 1997). It is believed that the actual timing of implantation of the embryo corresponds closely to the peak progesterone level (Tsubota et al. 1987). Although some studies contest this argument (Göritz et al. 1997), our results suggest that this may be the case in giant pandas because of the significant relationships we found between peak progesterone and subsequent fetus detection. The delay that we found in the peak progesterone level of the pregnant female giant pandas, accompanied by a relatively unvarying window between the time of peak progesterone to parturition, also supports previous studies done on single pregnant giant panda females (Chaudhuri et al. 1988, Monfort et al. 1989). If we assume that the giant panda embryo is implanted around the time the peak in urinary progesterone level occurs, then, according to our results, the period of delayed implantation in giant pandas is around $107.15 \pm 19.75$ days. 
Table 2 Fetal length $(\mathrm{cm})$ of two giant panda fetuses carried by pregnant females housed at the China Conservation and Research Center for the Giant Panda. Fetal length was measured using the measurement function of an ultrasound machine (Aloka SSD-900V ultrasound machine, 3.5 sector transducer).

\begin{tabular}{lcc}
\hline Year and female & Zhangka (2006) & Guoguo (2006) \\
\hline $\begin{array}{l}\text { Days until parturition } \\
20\end{array}$ & $-^{\mathrm{a}}$ \\
19 & $-^{\mathrm{a}}$ & \\
18 & & \\
17 & & \\
16 & & \\
15 & & \\
14 & 1.8 & 2.6 \\
13 & & 2.6 \\
12 & & 3.1 \\
11 & & 4.4 \\
10 & 7.4 & \\
9 & 7.4 & 6.6 \\
8 & 7.7 & 10.2 \\
7 & 10.1 & 10.8 \\
6 & & 11.6 \\
5 & & \\
4 & & \\
3 & & \\
1 & & \\
0 & & \\
\hline
\end{tabular}

${ }^{\mathrm{a}}$ Date of fetus detection.

In brown bears, peak serum progesterone levels are reached 60 days before parturition, but the fetus is not observed in the ultrasound until 33-35 days before parturition (Tsubota et al. 1987). In our study on giant pandas, the gap between peak progesterone level and fetus detection was shorter $(12.75 \pm 4.89$ days $)$ and the peak was reached closer to the time of parturition $(29.25 \pm 5.12)$. But the ratio between mean peak progesterone to total gestation length is similar in the two species $(60 / 225$ days $=0.27$ in brown bears and $29 / 124$ days $=0.24$ in pandas). Delayed implantation is believed to be an example of phylogenetic conservatism (Zhu et al. 2001), or a trait the panda has inherited long ago as a result of its ursid ancestry. The similarity in this ratio provides some degree of support for this theory. The shorter gestation length of giant pandas relative to other ursids may be related to the fact that giant pandas do not hibernate in winter and need to give birth to young earlier, so that they grow fur to survive the cold during winter months (Schaller et al. 1985). In addition, the implantation itself occurs during the time of year when their food is the most nutritious and may assure adequate fetal development (Schaller et al. 1985).

The gestation lengths observed in this study were similar in variability, but slightly shorter than those observed in the study on wild giant pandas (146.3 $\pm 11.3, N=6$, Zhu et al. 2001). This is similar to our more extensive records on gestation length of our larger population of captive giant pandas, for which we found a mean gestation length of $128.9 \pm 19.8$ days
$(N=73)$. More studies are needed to find out whether the difference in gestation length was a result of small sample size of wild individuals or whether there is some innate characteristic of the captive condition that caused this difference.

It is important to note that many of the females also mated naturally and the natural mating events (as opposed to the artificial insemination that followed them) could have resulted in pregnancy. Not all paternity has been determined at the time of this publication. Even for those in which paternity is known, many females mated with the same male on multiple days, leaving the actual date of conception unknown. Therefore, we standardized all pregnancies to start at the date of artificial insemination, the last possible date of conception. However, it should be noted that the maximum time frame that encompasses all natural mating events and artificial insemination is normally around 3 days, but is at most 5 days (so the duration of each period reported could be at most 5 days longer than indicated). More research is needed specifically on the effect of artificial insemination and its potential role in altering the duration of gestation.

\section{Description of giant panda fetal development}

Another novel finding in this study is the significant relationship between infant birth weight and length of the postfetal detection period (time between detection of fetus and birth). This relationship was significant even when controlling for the duration of total gestation period. This result suggests that while the duration of the period of embryonic dormancy may function in increasing the flexibility in the timing of birth, it does not play a significant role in dictating the amount of growth of the fetus. The relative importance of the fetal development phase may explain why the postfetal detection period is comparatively consistent in duration across individuals, despite the large variability in the length of the overall gestation period.

It is informative to discuss the results regarding fetus size in relationship to ultrasonography studies on other bears, particularly the well-studied brown bear. Brown bears have been first detected in ultrasound around 33-35 days before birth (Tsubota et al. 1987), which is nearly twice as early as giant pandas. Although our data are scant, the giant panda fetus is similar in size to the brown bear $(1.5-2.0 \mathrm{~cm})$ when first detected and appears to display a similar sigmoidal growth curve, where there is initially slow rate of growth, followed by rapid growth during the middle phase, only to slow at the end right before parturition (Tsubota et al. 1987).

The changes in female physiology coupled with the use of ultrasound provide useful information for detecting pregnancy in giant pandas, which has long been a difficult task in captive breeding and management of this species. At first glance, the narrow and relatively 
unvarying windows of peak progesterone level and swelling of mammary glands and vulva suggest that these signs could be good indicators for predicting pregnancy in this species. However, it should be noted that many of these signs are often observed in pseudopregnant females, such that further research is needed in order to be able to use these indicators to more precisely predict pregnancy. That being said, the short period of around 15-20 days between detection of the fetus and birth did not vary substantially among individuals, thus giving managers a reliable window in which they expect to detect a fetus in the ultrasound and subsequently plan for birth accordingly.

\section{Materials and Methods}

\section{Study subjects}

Nine adult female giant pandas (A. melanoleuca) were monitored in this study. Their ages ranged from 6 to 13 years old at the start of the study. Out of the nine pandas, four were monitored across two different breeding seasons during the period of 2005-2008. There were a total of 13 observed pregnancies. The pandas were housed in captivity at the China Conservation and Research Center for the Giant Panda (CCRCGP) in Wolong Nature Reserve, Sichuan, China, in captive enclosures $(11 \times 9 \mathrm{~m})$. They were encouraged to mate naturally with captive male pandas during the breeding season according to a protocol described in detail in Zhang et al. (2004). All female pandas were also artificially inseminated at their estimated peak estrus times so as to ensure pregnancy (see Howard et al. 2006). Peak estrus time was estimated by a combination of behavioral factors (increase in scent marking, vocalization, and lordosis) and physiological factors (change in vulval color and size, percentage cornified vaginal cells) (Zhang et al. 2004).

\section{Ultrasound imaging}

The adult females were monitored through B-mode ultrasound using an Aloka SSD-900V ultrasound machine (Aloka Co. Ltd, Wallingford, CT, USA), which was equipped with a 3.5 sector transducer. Ultrasounds began 1 month after breeding and continued once per month until swelling of the uterus was detected. After this point, ultrasounds were given at least once every 3 days. There were several females that received ultrasounds at a time when the fetus had already developed past the 3-day stage (when differentiation of the fetus occurs). These individuals were excluded from this paper. All pregnancies reported in Table 1 and throughout the paper were first detected at the earliest nondifferentiated phase. Nonetheless, the 3-day window of error should be noted.

The pandas lay on their backs in small cages during the examination and remained awake throughout. The animals in CCRCGP are accustomed to frequent medical checks and have been trained to lie still for examinations in exchange for food rewards. The panda's stomachs were shaved around the area of the uterus and coupling gel was applied. Once the uterus was located, an image was taken.

\section{Data collection and analysis}

For each pregnancy event, we recorded the date of artificial insemination, date of fetus detection, and date of parturition (birth of cub(s)). The prefetal detection period ran from the date of artificial insemination to the date of fetus detection. The postfetal detection period was between the date of parturition and the date of fetus detection. The duration of gestation was determined to be the time between insemination and parturition. In addition, we recorded the number of offspring produced in each parturition event and the date of parturition, and weighed the infants at the time of birth. (a)

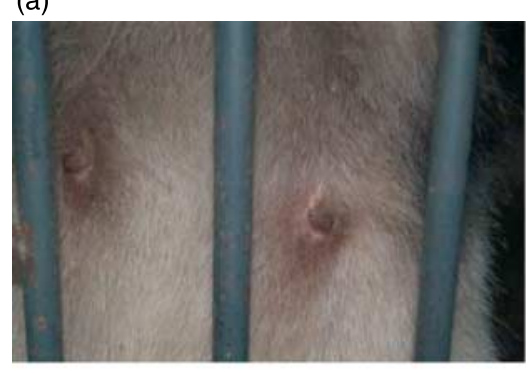

(c)

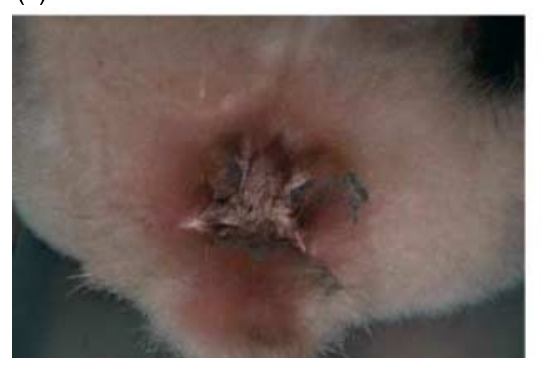

(b)

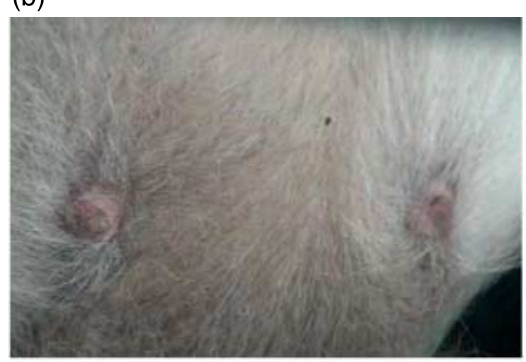

(d)

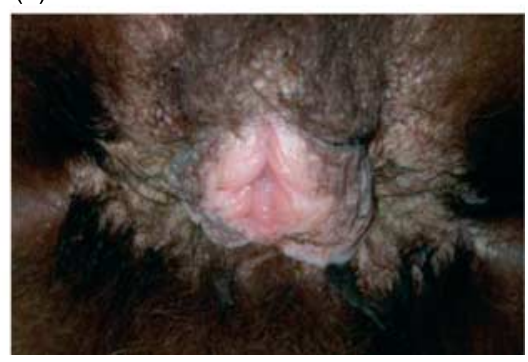

Figure $3(a-d)$ Images depicting swollen and unswollen mammary glands and vulva of pregnant female giant pandas at the China Conservation and Research Center for the Giant Panda (CCRCGP). Mammary glands pictured are those of Youyou while (a) unswollen - 60 days before giving birth in 2006 and (b) swollen - 22 days before giving birth in 2006. The vulva pictured is that of Feifei while (c) unswollen - 23 days before giving birth in 2006 and (d) swollen -2 days before giving birth in 2005. We present a 2006 image of Feifei's unswollen vulva (as opposed to 2005) because it was the best quality image to represent this state. 
We recorded observations on the development of the fetus and physiological changes in female pandas throughout the pregnancy. Owing to management constraints and individual animal sensitivities to different procedures, not all data were collected for every individual. Changes in the size of the mammary glands and vulva were determined according to a binary measure of $0 / 1$ (yes/no) swelling based on visual appearance and comparison of notes and photographs across days. We presented examples of these images in Fig. 3(a-d). We used the same system for detecting changes in the uterus, but decided to exclude these data from the analysis because they were overly subjective.

We also recorded basic qualitative observations about differentiation of the fetus such as appearance of head and limbs, differentiation of organs, and detection of heartbeat. We recorded length of the fetus (in $\mathrm{cm}$, measured from crown to rump) as detected in the ultrasound image when possible using the measurement function on the uterus machine. Owing to difficulties in obtaining a clear picture and human error, we were only able to obtain a series of multiple reliable estimates of fetus length over time in two individuals.

We monitored urinary progesterone levels of the adult females according to methods outlined by Czekala et al. (2003). Urine was collected in syringes from the cage floor approximately once per day. It was then placed in tubes in a freezer for storage. Pregnanediol-3-glucuronide (PDG) was measured in the samples using standard enzyme immunoassay protocol (Munro \& Stabenfeldt 1984). In this method, PDG antiserum (Munro, UC-Davis) was combined with PDG HRP (Munro, UC-Davis) and PDG standard (2.4-625 pg). TMB (Sigma) served as the substrate for the colorimetric endpoint change. The hormone concentration was indexed by $\mathrm{Cr}$ to control for differences in dilution of urine among samples. $\mathrm{Cr}$ was measured using a colorimetric assay (Taussky 1954). We presented results in units of mass $(\mathrm{ng}) / \mathrm{mg} \mathrm{Cr}$. It was logistically difficult to collect urine for all females included in this study; thus, we presented data from eight pregnant females only.

We conducted Pearson's product-moment correlation tests to analyze the relationship between date of fetus detection and dates of other signs of pregnancy, including swelling of the mammary glands, swelling of the vulva, and peak in urinary progesterone. We constructed an ordinary least-squares linear model to explain infant birth weight as a function of gestation characteristics, including age of the mother, number of offspring, length of total gestation period, and length of the period from fetus detection to parturition. This analysis was performed using the $\mathrm{R}$ software package ( $\mathrm{R}$ Development Core Team 2005).

\section{Declaration of interest}

There is no conflict of interest that could be perceived as prejudicing the impartiality of the research reported.

\section{Funding}

This work was supported by the Giant Panda International Collaborative Project Fund.

\section{Acknowledgements}

We gratefully acknowledge the dedicated efforts of the veterinary staff and animal keepers at the China Conservation and Research Center for the Giant Panda (CCRCGP) who worked tirelessly on data collection and animal care for this project. We also express our sincere thanks to Jianguo Liu for assisting with English translation and providing editorial suggestions to earlier versions of this manuscript.

\section{References}

Ballou JD, Miller PS, Xie Z, Wei R, Zhang H, Zhang A, Huang S, Sun S, David VA, O'Brien SJ et al. 2006 Analysis of demographic and genetic trends for developing a captive breeding masterplan for the giant panda. In Giant Pandas - Biology, Veterinary Medicine and Management, pp 495-519. Eds DE Wildt, A Zhang, H Zhang, DL Janssen \& S Ellis. Cambridge, MA: Cambridge University Press.

Chaudhuri M, Kleiman DG, Wildt DE, Bush M, Frank ES \& Thau RB 1988 Urinary steroid concentrations during natural and gonadotrophininduced oestrus and pregnancy in the giant panda (Ailuropoda melanoleuca). Journal of Reproduction and Fertility 84 23-28.

Czekala N, McGeehan L, Steinman K, Xuebing L \& Gual-Sil F 2003 Endocrine monitoring and its application to the management of the giant panda. Zoo Biology 22 389-400.

Göritz F, Hildebrandt T, Jewgenow K, Wagner N, Hermes R, Strauss G \& Meyer H 1997 Transrectal ultrasonographic examination of the female urogenital tract in nonpregnant and pregnant captive bears (Ursidae). Journal of Reproduction and Fertility Supplement 51 303-312.

Hildebrandt TB, Brown JL, Goritz F, Ochs A, Morris P \& SutherlandSmith M 2006 Ultrasonography to assess and enhance health and reproduction in the giant panda. In Giant Pandas - Biology, Veterinary Medicine, and Management, pp 410-439. Eds DE Wildt, A Zhang, H Zhang, DL Janssen \& S Ellis. Cambridge, UK: Cambridge University Press.

Hodges JK, Bevan DJ, Celma M, Hearn JP, Jones DM, Kleiman DG, Knight JA \& Moore HDM 1984 Aspects of the reproductive endocrinology of the female giant panda (Ailuropoda melanoleuca) in captivity with special reference to the detection of ovulation and pregnancy. Journal of Zoology 203 253-267.

Howard J, Huang Y, Wang P, Li D, Zhang G, Hou R, Zhang Z, Durrant BS, Spindler RE, Zhang $\mathbf{H}$ et al. 2006 Role and efficiency of artificial insemination and genome resource banking. In Giant Pandas - Biology, Veterinary Medicine, and Management, pp 469-494. Eds DE Wildt, A Zhang, H Zhang, DL Janssen \& S Ellis. Cambridge, MA: Cambridge University Press.

Lindburg DG, Czekala NM \& Swaisgood RR 2001 Hormonal and behavioral relationships during estrus in the giant panda. Zoo Biology 20 537-543.

McGeehan L, Li X, Jackintell L, Huang S, Wang A \& Czekala NM 2002 Hormonal and behavioral correlates of estrus in captive giant pandas. Zoo Biology 21 449-466.

Mead RA 1989 The physiology and evolution of delayed implantation in carnivores. In Carnivore Behavior, Ecology, and Evolution, pp 437-464. Ed. JL Gittleman. Ithaca, New York: Cornell University Press.

Monfort SL, Dahl KD, Czekala NM, Stevens L, Bush M \& Wildt DE 1989 Monitoring ovarian function and pregnancy in the giant panda (Ailuropoda melanoleuca) by evaluating urinary bioactive FSH and steroid metabolites. Journal of Reproduction and Fertility $\mathbf{8 5}$ 203-212.

Munro C \& Stabenfeldt G 1984 Development of a microtitre plate enzyme immunoassay for the determination of progesterone. Journal of Endocrinology 101 41-49.

R Development Core Team 2005 R: A Language and Environment for Statistical Computing, Vienna, Austria: R Foundation for Statistical Computing.

Sandell M 1990 The evolution of seasonal delayed implantation. Quarterly Review of Biology 6523. 
Schaller GB, Hu J, Pan W \& Zhu J 1985 The Giant Pandas of Wolong, Chicago, IL: University of Chicago Press.

Sutherland-Smith M, Morris PJ \& Silverman S 2004 Pregnancy detection and fetal monitoring via ultrasound in a giant panda (Ailuropoda melanoleuca). Zoo Biology 23 449-461.

Taussky HH 1954 A microcolorimetric determination of creatine in urine by the Jaffe reaction. Journal of Biological Chemistry 208 853-862.

Tsubota T, Takahashi Y \& Kanagawa H 1987 Changes in serum progesterone levels and growth of fetuses in Hokkaido brown bears. Bears. Their Biology and Management 7 355-358.

Zhang G, Zhang H, Chen M, He T, Wei R \& Mainka SA 1996 Growth and development of infant giant pandas (Ailuropoda melanoleuca) at the Wolong Reserve, China. Zoo Biology 15 13-19.
Zhang G, Swaisgood RR \& Zhang H 2004 Evaluation of behavioral factors influencing reproductive success and failure in captive giant pandas. Zoo Biology 23 15-31.

Zhu X, Lindburg DG, Pan W, Forney KA \& Wang D 2001 The reproductive strategy of giant pandas (Ailuropoda melanoleuca): infant growth and development and mother infant relationships. Journal of Zoology 253 141-155.

Received 11 June 2009

First decision 6 July 2009

Accepted 19 August 2009 